\title{
Shock-wave synthesis of alveolate graphene
}

\author{
Yazhu Lan ${ }^{1, a}$, Pengwan Chen ${ }^{1, b}$, Chunxiao $\mathrm{Xu}^{1, \mathrm{c}}$ and Jianjun $\mathrm{Liu}^{2, \mathrm{~d}}$ \\ ${ }^{1}$ State Key Laboratory of Explosion Science and Technology, Beijing Institute of Technology, \\ Beijing 100081, China; \\ ${ }^{2}$ Faculty of Science, Beijing University of Chemical Technology, Beijing 100029, China. \\ alanyazhu315@163.com, bpwchen@bit.edu.cn, 'c3120140441@bit.edu.cn, dijj-717@163.com
}

Keywords: shock wave, graphene, glocuse, RDX.

\begin{abstract}
In this paper, glucose and RDX as raw material, alveolate graphene was synthesized by shock wave in the detonation chamber. The results of TEM, HRTEM, SEM and XRD showed that alveolate graphene was synthesized in the wall of the detonation chamber. The ratio of RDX and glucose was an important role for the formation of honeycomb graphene, the high or low dosage was not in favor of the synthesis of the product, and moderate amount of RDX (3.2 g) which could provide a suitable temperature and pressure, promoted the synthesis of large area graphene sheets.
\end{abstract}

\section{Introduction}

Graphene is a kind of hexagonal honeycomb lattice of thin film, which is composed of carbon atoms with $\mathrm{sp}^{2}$ hybrid orbitals type and there is only one carbon atom thickness of 2 dimension (2 D) materials. In 2004, Novoselov and his partners discovered that monocrystalline graphitic (graphene) films were a few atoms thick but were nonetheless stable under ambient conditions, metallic, and of remarkably high quality [1]. Since then, more and more science researchers studied on it due to its unique physical and chemical properties. Alexander et al discovered that graphene can outperform carbon nanotubes in heat conduction [2]. Stoller et al have synthesized single-layer graphene which showed high electrical conductivity [3].

$\mathrm{Li}$ and his group undertook detailed scientific studies of large-area graphene films on copper substrates by chemical vapor deposition. The films were predominantly single-layer graphene, and they were continuous across copper surface steps and grain boundaries [4]. Sheng et al took use of melamine as the nitrogen source and the atomic percentage of nitrogen in doped graphene samples could be adjusted up to $10.1 \%$. This kind of NG in gram-scale could be used for electronic devices and cathodic materials for fuel cells and biosensors [5]. Stankovich et al discovered that reduction of exfoliated graphene oxide sheets in water with hydrazine resulted in graphene-based sheets with high-surface-area [6]. Wang et al took use of sodium-4-styrenesulfonate as an effective electrolyte, highly efficient and large-scale synthesis of graphene from graphite was produced by electrolytic exfoliation [7].

The shock wave action which characterizes by high temperature, high pressure, and a high strain rate lasting for a very short duration $\left(\sim 10^{-6} \mathrm{~s}\right)$ can induce various chemical reactions and phase transitions in materials. Shock waves have been successfully produced nano-sized pure carbon particles with both $\mathrm{sp}^{3}$ and $\mathrm{sp}^{2}$ bonded structures. David et al have measured that graphite with sufficient orientational order transformed to a diamond-like phase by shock-induced martensitic transformation [8]. Multi-walled carbon nanotubes (CNTs) have been efficiently synthesized by a self-heating detonation process. Among these tubes, some of them were well crystallized but others with lots of structural defects, especially for the tubes with thin walls and bamboo-like shapes. This approach suggested that it was possible for a large-scale synthesis of CNTs under high-density and high-pressure conditions [9]. Onion-like carbon has been synthesized by detonation-assisted chemical vapor deposition. The OLC contained about 7-10 closed shells and exhibited a narrow distribution of 4-6 nm. It was also suggested that the detonation-assisted chemical vapor deposition was a very simple and efficient technology to synthesize pure and small OLC [10]. Yin et al have synthesized nitrogen doped graphene by shock wave loading, which using calcium carbonate, 
magnesium and ammonium nitrate as the raw materials [11]. In this paper, by using glucose as carbon source, we heated RDX to detonate to produce shock waves to synthesize alveolate graphene.

\section{Experiment}

In the study, glucose was used as carbon source, $\mathrm{RDX}$ was heated to detonate to provide shock wave. Both the raw materials were AR. Before the detonation experiments, the starting materials were mixed manually in mild way, then pressed the mixture into cylinder by press machine. The cylinder of raw materials was performed in a $100 \mathrm{ml}$ detonation chamber to $10 \mathrm{kPa}$ vacuum atmosphere, and induced by external heating (with a heating rate of $15{ }^{\circ} \mathrm{C} / \mathrm{min}$ ) to approximately $150{ }^{\circ} \mathrm{C}$, the RDX detonated thermally, which caused a sudden temperature rise (almost $70{ }^{\circ} \mathrm{C}$ ) recorded by a temperature sensor [12]. After the detonation, the chamber was naturally cooled down to the room temperature. The gaseous product was vented. The black solid product was uniformly dispersed in the diluted hydrochloric acid by ultrasonic for an hour. After that, distilled water was used to wash it to neutral. The experimental formula are shown in table 1, the difference between experiment 1 and experiment 2 was that the cylinder of 1 placed into the detonation chamber directly, and the cylinder of 2 was wrapped by copper [4], then placed into the detonation chamber.

Table1 Experimental formula

\begin{tabular}{ccc}
\hline No. & The ratio of raw materials & Remark \\
\hline 1 & 2.4 g RDX +0.6 g glucose & Naked cylinder \\
2 & 2.4 g RDX +0.6 g glucose & Cylinder wrapped by Cu \\
3 & 1.6 g RDX +0.4 g glucose & Naked cylinder \\
4 & 3.2 g RDX +0.8 g glucose & Naked cylinder \\
\hline
\end{tabular}

The microstructure of the product was observed using a transmission electron micro-scope (TEM) (Tecnai 20) and high resolution TEM (HRTEM), which was equipped with energy dispersive X-ray spectrometer (EDX) for elemental analysis. Scanning electron microscopy (SEM) (Japan's Hitachi $S$ - 4800) was also used to observe the microstructure of the product. An X-ray diffractometer (XRD) (Rigaku D/MAX-2500) with $\mathrm{Cu} \mathrm{K \alpha}$ radiation $(\lambda=0.15406 \mathrm{~nm}$ ) was used to determine the phase composition of the products at a work voltage of $40 \mathrm{kV}$ and a work current of $200 \mathrm{~mA}$.

\section{Result and discussion}

The formation of graphene is largely affected by the deposition basement. Therefore, we design the experiments of No. 1and No. 2. High resolution TEM images of No. 1 and No. 2 are shown in Fig. 1, and the inset of (a) and (b) show SEAD pattern. From the pictures, we can see that No. 1 is better crystalline than No. 2, and the crystallization of No. 1 is hexagonal system. Fig. 2 shows typical TEM images of No. 2, (a) is before the cleaning of hydrochloric acid, (b) is after the cleaning of hydrochloric acid, (c) is the EDS spectrum of (a), (d) is the EDS spectrum of (b). From the images, we discover that the cleaning with hydrochloric acid doesn't destroy the structure of the graphene, and the impurities can be cleaned out. From the image (c), the impurities of No. 2 are composed of iron, manganese, nickel and so on, which are the same as the component of the chamber. In this situation, the deposition basement of No. 2 is also the chamber wall rather than the copper. Furthermore, the better crystalline is the sample of No. 1. Hence, we decide to choose the condition of No. 1 to take the following experiments.

In the reaction process, temperature and pressure play important roles. So the dosage of RDX is an important variable during the reaction. According to this fact, we design the experiments of No. 1, No. 3 and No. 4. Fig. 2 shows the typical XRD patterns of No. 1, No. 3 and No. 4. All the patterns show a broad peak around $26.0^{\circ}$ which can be ascribed to the (002) plane of graphitic carbon [13]. From the XRD patterns, the best crystallization is No. 1. 


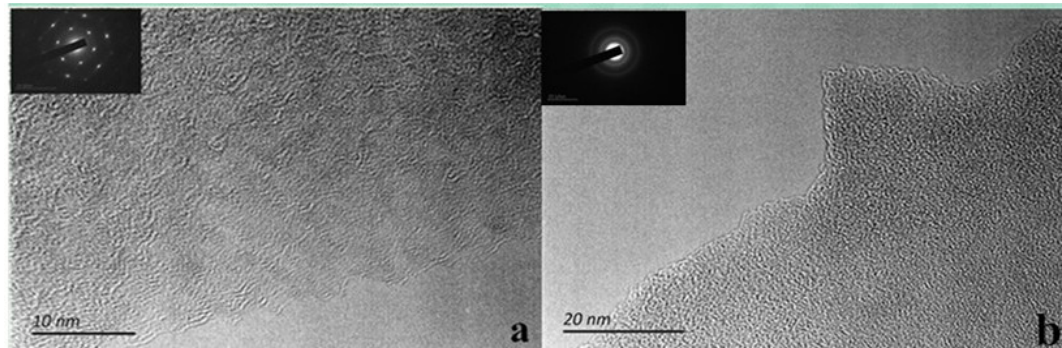

Fig. 1 HRTEM images of No. 1 (a) and No. 2 (b). The inset of (a) and (b) show SEAD pattern.

Fig.4-6 show typical SEM and TEM images of the samples of No. 1, No. 3 and No. 4 . All of the samples have been consisted of alveolate graphene. Among these samples, No. 1 has the largest scale in the area with fewer layers, the morphology of the product of No. 3 or No. 4 was incomplete with more layers.
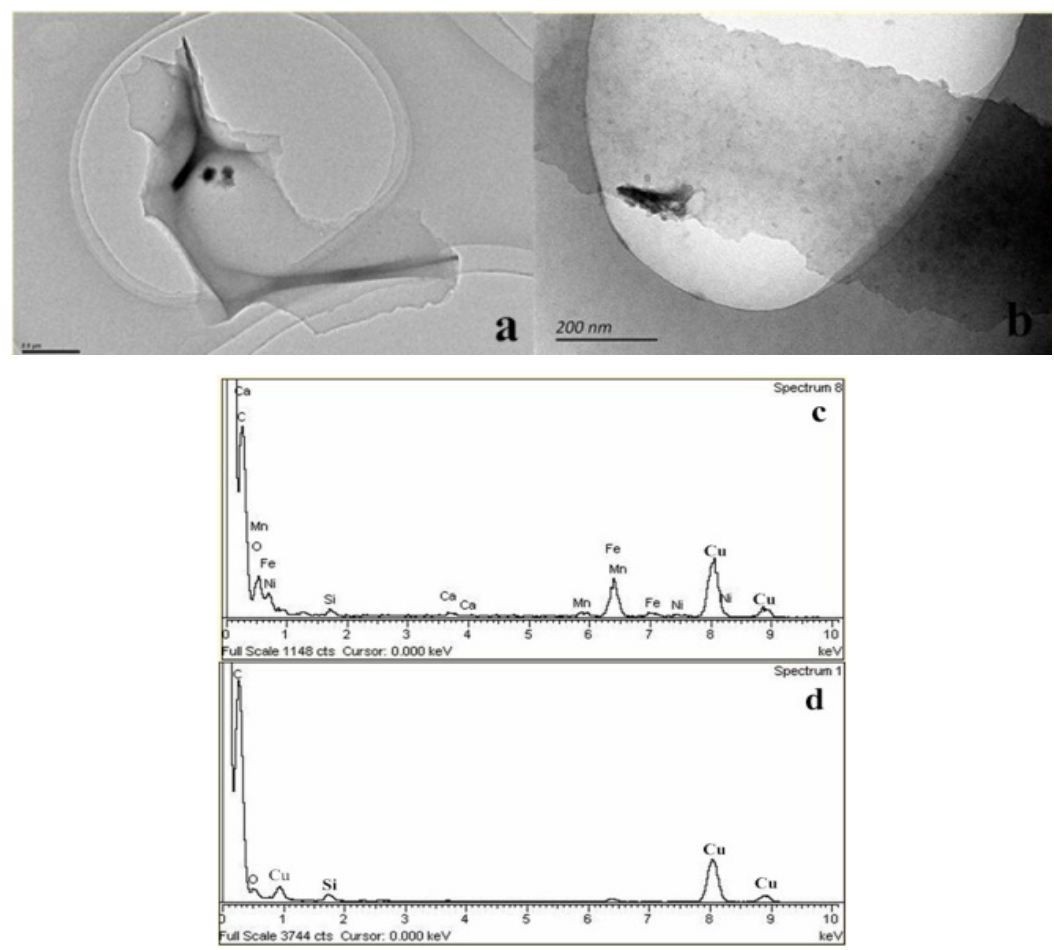

Fig. 2 Typical TEM images of No. 2, (a) is before the cleaning of hydrochloric acid, (b) is after the cleaning of hydrochloric acid, (c) is the EDS spectrum of (a), (d) is the EDS spectrum of (b)

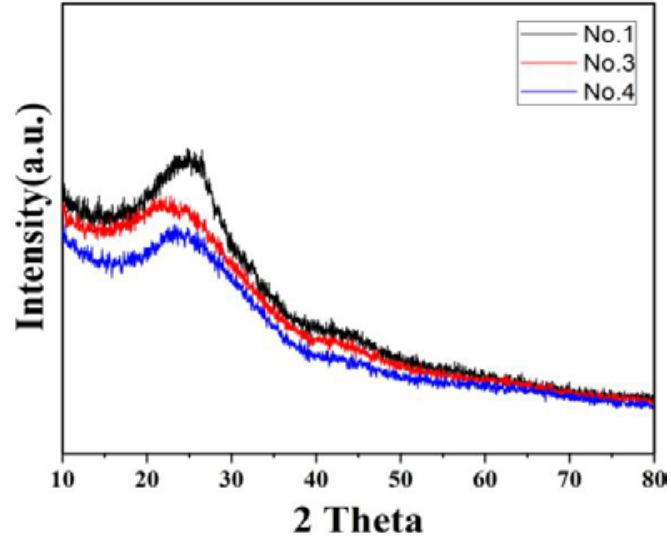

Fig. 3 Typical XRD patterns of No. 1, No. 3 and No. 4

According to the analysis, we have synthesized alveolate graphene by shock wave from glucose successfully. During the explosion, lots of heat and shock wave generate instantaneously, and the glucose decomposes into carbon atoms, and then conglomerates to carbon drops, the drops encounter the chamber wall to generate the alveolate graphene [14]. Combine with the result of the XRD patterns, the images of SEM and TEM, we discover that the graphene with large scale, good crystalline and thin sheet have been got from the medium dosage of RDX (2.4 g). Maybe the low 
dosage of RDX (1.6 g) can't provide enough energy so that the raw materials can't decompose completely, leading to the incomplete structure of alveolate graphene. When the dosage of RDX is high (3.2 g), the pressure in the chamber is high too. And the decomposition of glucose is violent so as to the more layers of graphene.

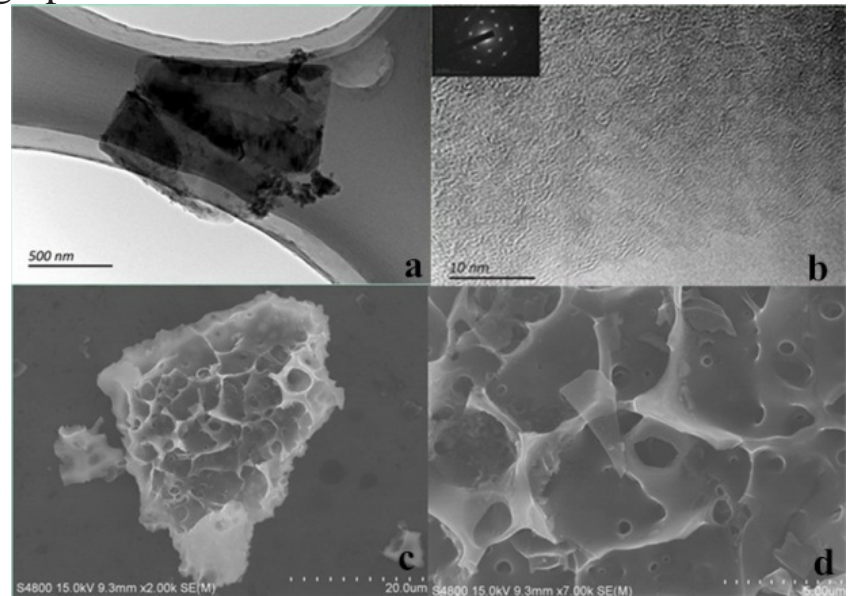

Fig. 4 Typical TEM and SEM images of No. 1
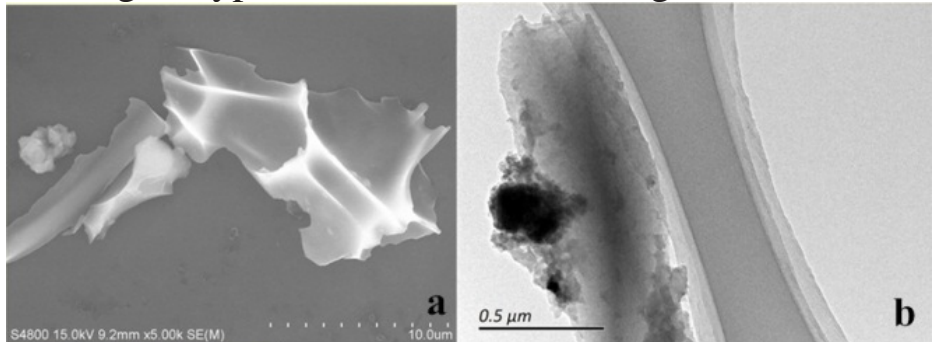

Fig. 5 Typical SEM and TEM images of No. 3
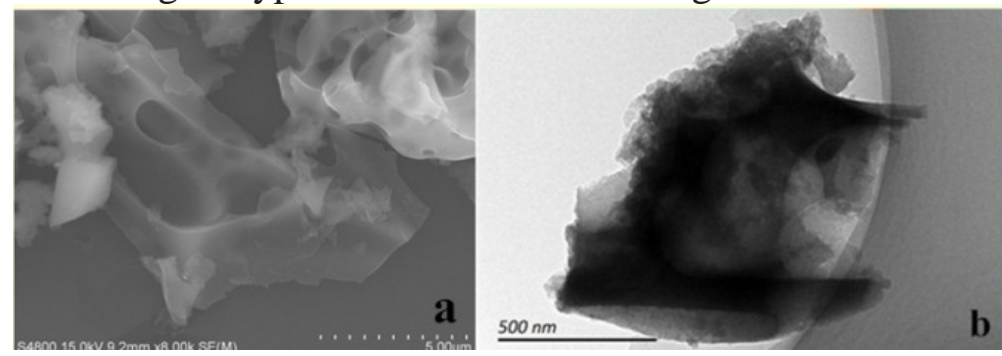

Fig. 6 Typical SEM and TEM images of No. 4

\section{Conclusion}

In this work, alvaolate graphene has been produced by detonation of RDX and glucose mixture with different dosage of RDX. The deposition basement for graphene plays an important role and the chamber wall is a good choice. The dosage of RDX is crucial for the formation of alvaolate graphene. The high (3.2 g) and low (1.6 g) dosages are not in favor of the synthesis of alvaolate graphene.

\section{Acknowledgments}

The authors would like to express their thanks for the financial support of National Natural Science Foundation of China under Grant Nos. 11221202.

Address correspondence to Pengwan Chen, State Key Laboratory of Explosion Science and Technology, Beijing Institute of Technology, Beijing, China. E-mail: pwchen@bit.edu.cn.

\section{References}

[1]. K. S. Novoselov, A. K. Geim, S. V. Morozov, Electric Field Effect in Atomically Thin Carbon 
Films, J. Science. 306. 5696 (2004) 666-669.

[2]. Alexander A. Balandin, Suchismita Ghosh, Wenzhong Bao, Superior Thermal Conductivity of Single-Layer Graphene, J. Nano Letters. 8. 3 (2008) 902-907.

[3]. Stoller Meryl D., Park Sungjin, Zhu Yanwu, Graphene-Based Ultracapacitors, J. Nano Letters. 8. 10 (2008) 3498-3502.

[4]. Xuesong Li, Weiwei Cai, Jinho An, Large-Area Synthesis of High-Quality and Uniform Graphene Films on Copper Foils, J. Science. 324. 5932 (2009) 1312-1314.

[5]. Zhen-Huan Sheng, Lin Shao, Jing-Jing Chen, Catalyst-Free Synthesis of Nitrogen-Doped Graphene via Thermal Annealing Graphite Oxide with Melamine and Its Excellent Electrocatalysis, J. ACS Nano. 5. 6 (2011) 4350-4358.

[6]. Stankovich Sasha, Dikin Dmitriy A., Piner Richard D, Synthesis of graphene-based nanosheets via chemical reduction of exfoliated graphite oxide, J. Carbon. 45.7 (2007) 1558-1565.

[7]. Wang Guoxiu, Wang Bei, Park Jinsoo, Highly efficient and large-scale synthesis of graphene by electrolytic exfoliation, J. Carbon. 47. 14 (2009) 3242-3246.

[8]. Erskine D J, W.J. Nellis, Shock-induced martensitic transformation of highly oriented graphite to diamond, J. Journal of Applied Physics. 71. 10 (1992) 4882.

[9].Lu Y, Z Zhu, Z Liu, Catalytic growth of carbon nanotubes through CHNO explosive detonation, J. Carbon. 42.2 (2004) 361-370.

[10]. Du J., R. Zhao, Z. Zhu, A facile approach for synthesis and in situ modification of onion-like carbon with molybdenum carbide, J. Physics status solidi (a). 208. 4 (2011) 878-881.

[11]. Yin Hao, Chen Pengwan, Xu Chunxiao, Shock-wave synthesis of multilayer graphene and nitrogen-doped graphene materials from carbonate, J. Carbon. 94 (2015) 928-935.

[12]. Yin Hao, Gao Xin, Xu Chunxiao, Detonation Synthesis of Carbon-Encapsulated Magnetic Nanoparticles, J. Fullerenes Nanotubes and Carbon Nanostructures. 23.7 (2015) 605-611.

[13]. Zhang Geng, Lu Wangting, Cao Feifei, N-doped graphene coupled with Co nanoparticles as an efficient electrocatalyst for oxygen reduction in alkaline media, J. Journal of Power Sources. 302 (2016) 114-125.

[14]. Du Guixiang, Feng Shouai, Zhao Jianghong, Particle-Wire-Tube Mechanism for Carbon Nanotube Evolution, J. Journal of the American Chemical Society. 128. 48 (2006) 15405-15414. 